\title{
INTERVENCIONES EN LOS MONUMENTOS HISTÓRICOS DEL CENTRO DE LIMA: UN ANÁLISIS DE LA METODOLOGÍA APLICADA EN LA CAMPAÑA ADOPTE UN BALCÓN ${ }^{[\times]}$
}

\author{
INTERVENTION IN THE HISTORICAL MONUMENTS OF THE CENTER OF LIMA: \\ AN ANALYSIS OF THE METHODOLOGY APPLIED IN THE ADOPT A BALCONY CAMPAIGN \\ MARÍA COPAIRA ORTIZ ${ }^{\left({ }^{(*)}\right)}$ \\ Fecha de recepción: 06 de abril de 2015 \\ Fecha de aprobación: 27 de abril de 2015
}

\section{RESUMEN}

El Centro Histórico de Lima es uno de los principales elementos de identidad de la ciudad, por lo cual su conservación y revitalización debería ser objeto de atención especial. Pese a ello, durante mucho tiempo, ha sido enterrado en el olvido; ha subsistido en total abandono, lo cual ha generado su degradación paulatina. Sin embargo, durante los últimos años han tenido lugar múltiples intervenciones en distintas edificaciones en esta zona de la ciudad. Lamentablemente, algunas de estas intervenciones han puesto en peligro su autenticidad, pues los planteamientos y "criterios" aplicados en dichos procesos son de diversa índole: están basados en conceptos teóricos diferentes extraídos de las distintas interpretaciones dadas a las teorías de intervención y las numerosas cartas de salvaguarda patrimonial. Es de vital importancia reflexionar sobre las consecuencias de las diferencias en la interpretación y aplicación de los conceptos planteados en las teorías y cartas, pues ello permitirá analizar los procesos de intervención con la finalidad de hacer recomendaciones y/o establecer criterios que mejoren el manejo de dichos procesos e incorporen el uso de modelos de gestión patrimonial. En el siguiente texto se planteará una metodología de intervención, que se analizará a partir del caso concreto de la campaña Adopte un Balcón, emprendida por la Municipalidad Metropolitana de Lima entre los años 1997 y 2002.

\section{PALABRAS CLAVE}

Centro Histórico de Lima, edificaciones patrimoniales, intervenciones

\begin{abstract}
The Historic Center of Lima is one of the main elements of the city's identity, which is why its sustainability and revitalization should be given special attention. Nevertheless, for a long time it has been buried in oblivion, subsisting in a state of total abandonment that has led to its gradual degradation. However, in recent years multiple interventions in the buildings in that area have taken place, all aiming to safeguard them. On the other hand, some of those interventions have actually endangered the building's authenticity, because the planning and "criteria" employed in developing the project are based on different theoretical concepts resulting from the interpretations of various theories of intervention and numerous letters of patrimonial preservation. It is vital to reflect on the consequences of the application of the interpretations given to this theories and letters, because that will allow the analysis of intervention processes in order to establish recommendations and suggest criteria that will improve the management of these processes and incorporate the use of patrimonial management models. So, here an intervention methodology is raised and analyzed using as an example the specific case of the Adopt a Balcony campaign, undertaken by the Metropolitan Municipality of Lima between 1997 and 2002.
\end{abstract}

\section{KEYWORDS}

Historic Center of Lima, patrimonial buildings, interventions

(*) El presente artículo forma parte de la investigación titulada “¿Conservar, restaurar, intervenir? Del INC al Ministerio de Cultura. Intervenciones en el Centro Histórico de Lima (1970-2010)" desarrollada en el Taller de Investigación en Historia de la Arquitectura 1 y 2 (2010) a cargo del Prof. Mg. Arq. José Beingolea Del Carpio y el Prof. Mg. Arq. Carlos Enrique Guzmán García, en la Facultad de Arquitectura, Urbanismo y Artes de la Universidad Nacional de Ingeniería.

${ }_{(* *)}^{*}$ María Copaira Ortiz es bachiller en Arquitectura por la Facultad de Arquitectura, Urbanismo y Artes de la Universidad Nacional de Ingeniería, ha realizado diversos estudios sobre temática patrimonial, Curso de Preservación, Conservación y reciclaje del Patrimonio Histórico Urbano y Rural - AECID (2012). Entre otros, ha participado en diversos proyectos de intervención en el patrimonio edificado de Lima y actualmente se encuentra cursando la Maestría en Arte Peruano y Latinoamericano con mención en Historia del Arte en la Facultad de Letras y Ciencias Humanas de la Universidad Nacional Mayor de San Marcos. 
La intervención en el patrimonio edificado de los centros históricos es un tema que desde hace mucho tiempo ha generado gran interés, por lo cual sigue siendo objeto de polémica y debate entre historiadores, arqueólogos, arquitectos, urbanistas, economistas, diversos especialistas y el público en general, debate que ha sido motivo de una serie de publicaciones sobre teorías de restauro arquitectónico, metodologías de conservación, criterios de intervención e, incluso, textos internacionales de legislación sobre la salvaguarda del patrimonio monumental, las cartas internacionales de restauración.

La difusión de estas cartas dio origen a los discursos que aún hoy en día siguen siendo hegemónicos en nuestro centro histórico; estos abarcan desde posturas demoledoras de renovación urbana mediante el reemplazo de edificaciones antiguas con obra nueva hasta juicios de tipo netamente conservacionista. Estas posturas no son sino consecuencia de una lectura y aplicación las cartas internacionales que no da importancia al hecho de que la mayoría de ellas son producto de un pensamiento predominantemente eurocentrista y fueron concebidas en contextos culturales, políticos, económicos y/o históricos muy diferentes al local. Debido a esta discrepancia, determinar el alcance de dichas intervenciones y legitimar la acción sobre el legado arquitectónico todavía es una tarea pendiente, debido también en un sinnúmero de casos a la falta o al mal manejo de planes de gestión patrimonial que deberían acompañar a los proyectos de intervención.

Las cuatro principales causas de esta problemática en los criterios de intervención y en el adecuado uso de un plan de gestión radican en los siguientes factores generales:

- Las bases de datos existentes respecto a las edificaciones patrimoniales pertenecientes al Centro Histórico de Lima en la mayoría de casos son inexistentes, se encuentran incompletas y/o están desactualizadas.

- No existe una legislación adecuada para el manejo y protección del patrimonio en el Perú. Como consecuencia de ello, se recurre a normas de acción de carácter internacional (cartas internacionales de restauración). ${ }^{1}$

- No existe aún un manejo consciente e integral de modelos de gestión patrimonial en el medio, que permita un desarrollo sostenible y garantice la conservación del bien intervenido. Son pocos los proyectos realizados que han contado con un plan de gestión durante su proceso.

- Quizás el principal factor que afecta la práctica de las intervenciones es el mal uso del término criterio, que la Real Academia Española (2011) define como "norma para conocer la verdad / juicio o discernimiento" (p. 1710). Frente a la ausencia de legislación sobre intervenciones, el profesional encargado del proyecto es quien define los criterios de intervención, basándose en opiniones o valoraciones sobre la restauración que se fundamentan solo en interpretaciones personales. Sin embargo, se debería entender el termino criterio no como una opción de gustos personales o de vínculo a alguna escuela, sino como un sistema de investigación y deducción, pues estas diversas interpretaciones, hechas sin rigor científico, generan conflictos al momento de plantear una intervención².

\footnotetext{
1. Si bien en cierto que existe la Norma A.140 del Reglamento Nacional de Edificaciones y el Reglamento de la Administración del Centro Histórico de Lima - Ordenanza № 062, se trata de normas muy generales que contemplan lineamientos base, pero no cuentan con el rigor necesario para un manejo adecuado de las intervenciones, pues dejan muchos vacíos en cuanto al tratamiento de la edificación en sí; solo contemplan criterios que se centran principalmente en la conservación del perfil urbano y la zonificación de usos de suelo.

2. Resulta interesante en este punto citar a Ignasi de Solá-Morales (2006), quien otorga al concepto de intervención dos acepciones. La primera en un sentido general, pues "cuando hablamos de intervención debería entenderse cualquier tipo de actuación que puede hacerse en un edificio o en una arquitectura. Las actuaciones que sean de restauración, de defensa, de preservación, de conservación, de reutilización, etc., todas ellas podrían ser designadas con un término máximamente general que sería justamente el de intervención" (p. 15). En segundo lugar, está el significado más restringido y especifico, que hace referencia a la existencia de un conflicto de interpretación, pues "todo problema de intervención es siempre un problema de interpretación de una obra de arquitectura ya existente, porque las posibles formas de intervención que se plantean siempre son formas de interpretar el nuevo discurso que el edificio puede producir" (Ignasi de Solá-Morales, 2006, p. 15).
} 
Los referidos factores generales que condicionan nuestro medio son la fuente de diversas interrogantes a partir de las cuales en este texto se busca determinar cómo se han llevado a cabo las intervenciones en los bienes culturales inmuebles del Centro Histórico de Lima. El punto de partida es la identificación de las principales influencias teóricas que han tenido relevancia en el Perú, así como la determinación de los factores que se toman en cuenta en el planteamiento de toda intervención. Dicho análisis se realizó mediante la evaluación de las principales intervenciones realizadas desde la década de 1970 hasta el año 2010, periodo en el cual tuvieron lugar puntos importantes de inflexión, como la creación del Instituto Nacional de Cultura (INC) en el año 1972 y su posterior desarticulación, y la creación del Ministerio de Cultura (MINCULT) en el año 2010.

Para realizar el análisis de casos ha sido de vital importancia encontrar y definir cuáles son los diversos factores que deben ser tomados en cuenta al momento de plantear y ejecutar un proyecto de intervención. Para encontrar esos factores se ha tomado como punto inicial la clasificación de los procesos de ejecución de intervenciones en dos categorías: el proceso integral y el proceso parcial.

La Real Academia Española (2011) define el término integral como aquello que es "dicho de cada una de las partes de un todo: Que entra en su composición sin serle esencial, de manera que el todo puede subsistir, aunque incompleto, sin ella" (p. 3221). A partir de dicha acepción se ha definido el proceso integral como aquel en el cual se toma en cuenta todos los factores que afectan a la edificación, tanto de modo directo como indirecto, para plantear un proceso de intervención adecuado, que integre cada uno de ellos a través de un proyecto de gestión de patrimonio.

El término parcial, según la Real Academia Española (2011), indica que algo es "Perteneciente o relativo a una parte del todo" (p. 4177). Se ha definido, en base a este concepto, el proceso parcial como aquel en el cual no se han tomado en cuenta todos los factores que afectan la vida de la edificación, sino únicamente la intervención en la materia del mismo, dejando de lado muchos factores importantes que al ser obviados en el proceso de intervención posteriormente son causa del deterioro de la edificación.

Estos tipos de procesos fueron analizados subdividiéndolos en tres etapas (Gráfico 1):

- La etapa de preintervención como antecedente para definir la evolución histórica, y el valor cultural y arquitectónico de la edificación.

- La etapa de intervención, en la cual se debe garantizar que la intervención realizada sea adecuada, según lo establecido en los estudios previos realizados en la primera etapa.

- La etapa posintervención, en la que por medio de un plan de gestión que contemple los diversos factores se garanticen la conservación del inmueble y su sostenibilidad a través del tiempo.

Cada una de estas etapas, a su vez, se relaciona de modo directo con otros tipos de factores que se engloban en este estudio en dos grandes grupos, ya que intervienen en la conservación o deterioro de los bienes monumentales inmuebles:

- Factores internos: Aquellos que son inherentes al edificio, como su estado de conservación o deterioro, los materiales usados, el estilo arquitectónico, la funcionalidad, la originalidad de las partes, el grado de alteración, etc.

- Factores externos: Aquellos que afectan de modo indirecto a la edificación patrimonial, como el entorno urbano y diversos factores sociales (como la tugurización), políticos (como los cambios de usos del suelo) y económicos (como la especulación en el valor del suelo), etc. 
devenir VoL. 2, №3, ENERO - JUNIO 2015, PP. 63-78 - ESTUDIOS I ISSN 2312-7570

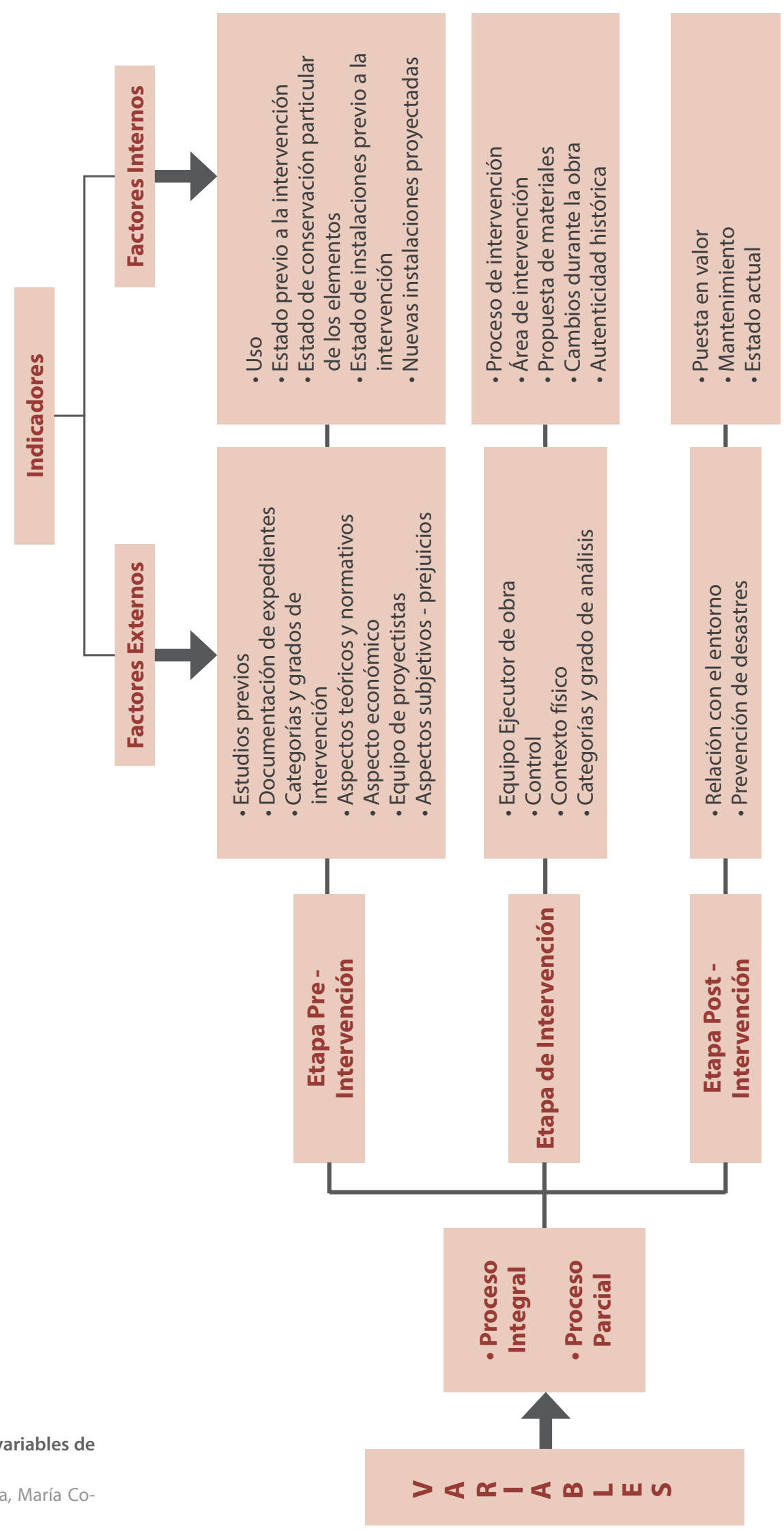
análisis.

Fuente. Elaboración propia, María Copaira, 2015. 
Estos factores se relacionan con las tres etapas señaladas de la siguiente manera:

Factores externos en la etapa de preintervención:

- Los estudios previos: Aquellos estudios realizados inicialmente en la edificación que será intervenida, que derivan en documentación fundamental para el planteamiento y desarrollo del proyecto de intervención.

- La documentación de los expedientes: Los expedientes del inmueble que han sido usados para la aprobación del proyecto, y la tramitación de licencias y/o permisos ante las entidades correspondientes (La Municipalidad de Lima y el Ministerio de Cultura).

- Las categorías y grados de Intervención³: La definiciones y limitaciones aplicadas por el Instituto Nacional de Cultura (posteriormente Ministerio de Cultura) a la intervención, de acuerdo al proyecto presentado, a los conceptos y procedimientos que se estipulan en las cartas internacionales, y las normas y reglamentación nacional vigente.

- Los aspectos teóricos y normativos: Las teorías de intervención, y la normativa existente y vigente en su coyuntura histórica, y en relación a la postura adoptada en la intervención.

- El aspecto económico: El valor monetario o costo que implicó el proceso de intervención, factor que condiciona crucialmente la intervención.

- El equipo de proyectistas: Quiénes plantearon y realizaron el proyecto de intervención, con qué experiencia y conocimiento contaban sobre los procesos de intervención en patrimonio edificado, y cuál era su filiación teórica o tendencia, si la tuviesen.

- Los aspectos subjetivos o prejuicios: Aquellas opiniones previas, por lo general desfavorables (Real Academia Española, 2011), basadas en interpretaciones personales y subjetivas de las recomendaciones generales, que influyen en qué criterios son tomados en cuenta para el desarrollo y ejecución de un proyecto de intervención.

Factores externos en la etapa de intervención:

- El equipo ejecutor de obra: El equipo que lleva a cabo el proyecto planteado, y la experiencia y conocimiento que tenían sobre los procesos de intervención en patrimonio edificado al momento de ejecutar la obra.

- Control: El control y seguimiento de la obra de parte de todos los actores (Residente, supervisor, Instituto Nacional de Cultura, Municipalidad de Lima, etc.), y su calidad.

- El contexto físico: La accesibilidad a la obra y la seguridad en la zona.

- Las categorías y el grado de intervención: Se vuelven a medir de acuerdo al desarrollo de la obra, pues siempre surgen replanteos en el camino.

Factores externos en la etapa de posintervención:

- La relación con el entorno: El nuevo uso y la expresión formal de la obra.

\footnotetext{
3. El grado de intervención está definido de la siguiente manera según documentación oficial del MINCULT: Para más detalle revisar: http://www.cultura.gob.pe/patrimonio

Grado 1: Permite la conservación y restauración de los componentes estructurales arquitectónicos, ornamentales y de carpintería y su adecuación a nuevo uso.

Grado 2: Permite, además de lo indicado en el grado 1, la remodelación siempre y cuando se mantenga las características del mismo inmueble.

Grado 3: Permite la rehabilitación del inmueble mediante el uso de tecnología constructiva contemporánea. Grado 4: Permite realizar intervención incluso de demolición siempre y cuando la obra nueva que se proyecta se integre adecuadamente al contexto urbano.

Grado 5: Este grado se asigna a áreas baldías dentro del inmueble o manzana, en las cuales las obras nuevas deberán integrarse al contexto urbano.
} 
- Prevención de desastres: Acciones tomadas para prevenir un desastre que pudiera destruir el bien patrimonial en cuestión.

Factores internos en la etapa de preintervención:

- El uso: El uso original y al propuesto para el rescate del inmueble, lo cual influye de modo radical en la conservación del bien intervenido.

- El estado previo a la intervención: La situación del inmueble antes de su intervención, cuyo análisis es útil para realizar una comparación una vez finalizada la obra.

- El estado de conservación particular de elementos: Estado previo a la intervención, más detallado, que se categoriza de tres maneras, de acuerdo con las siguientes definiciones:

Bueno: "adj. Dicho de una cosa: No deteriorada y que puede servir". (Real Academia Española, 2011, p. 909).

Regular: "adv. m. Medianamente, no demasiado bien". (Real Academia Española, 2011, p. 4792).

Malo: "adj. Deslucido, deteriorado." (Real Academia Española, 2011, p. 3559).

- El estado de instalaciones previo a la intervención: Este factor se calificará con las mimas condiciones anteriormente mencionadas (bueno - malo - regular).

- Nuevas instalaciones proyectadas: Modificaciones o ampliación de las redes existentes.

Factores internos en la etapa de intervención:

- El proceso de intervención: Cada una de las etapas que componen el proceso de intervención.

- El área de intervención: El porcentaje de área intervenida sobre el total del área de la unidad inmobiliaria.

- La propuesta de materiales: El uso de materiales nuevos y/o originales en los diversos elementos constructivos del inmueble, y su diferenciación de los elementos originales.

- Los cambios durante la obra: Las modificaciones que surgen in situ y cómo son subsanadas.

- La autenticidad histórica: Valoración que se realiza mediante la medición del porcentaje de área de los elementos originales, perdidos, añadidos y/o modificados.

Factores internos en la etapa de posintervención:

- La puesta en valor: Según los estipulado en las Normas de Quito (1967) “Poner en valor un bien histórico o artístico equivale a habitarlo en las condiciones objetivas y ambientales que, sin desvirtuar su naturaleza, resalten sus características y permitan su óptimo aprovechamiento" (punto Vl, ítem 2).

- El mantenimiento: El cuidado que debe dársele al edificio una vez que la intervención esté finalizada, pues ello garantiza que la edificación se conserve en buen estado durante mayor tiempo

- El estado actual: El estado posterior al proceso de intervención.

En la mayoría de casos revisados se ha podido observar que no se han tomado en cuenta los factores externos, pues los procesos de intervención se centran solo en la materia del edificio, y se dejan de lado los demás factores, lo cual genera dificultades en lo que respecta a su sostenibilidad y conservación. Esta carencia es consecuencia de la falta de aplicación de modelos de gestión que integren dichos factores al proceso. 


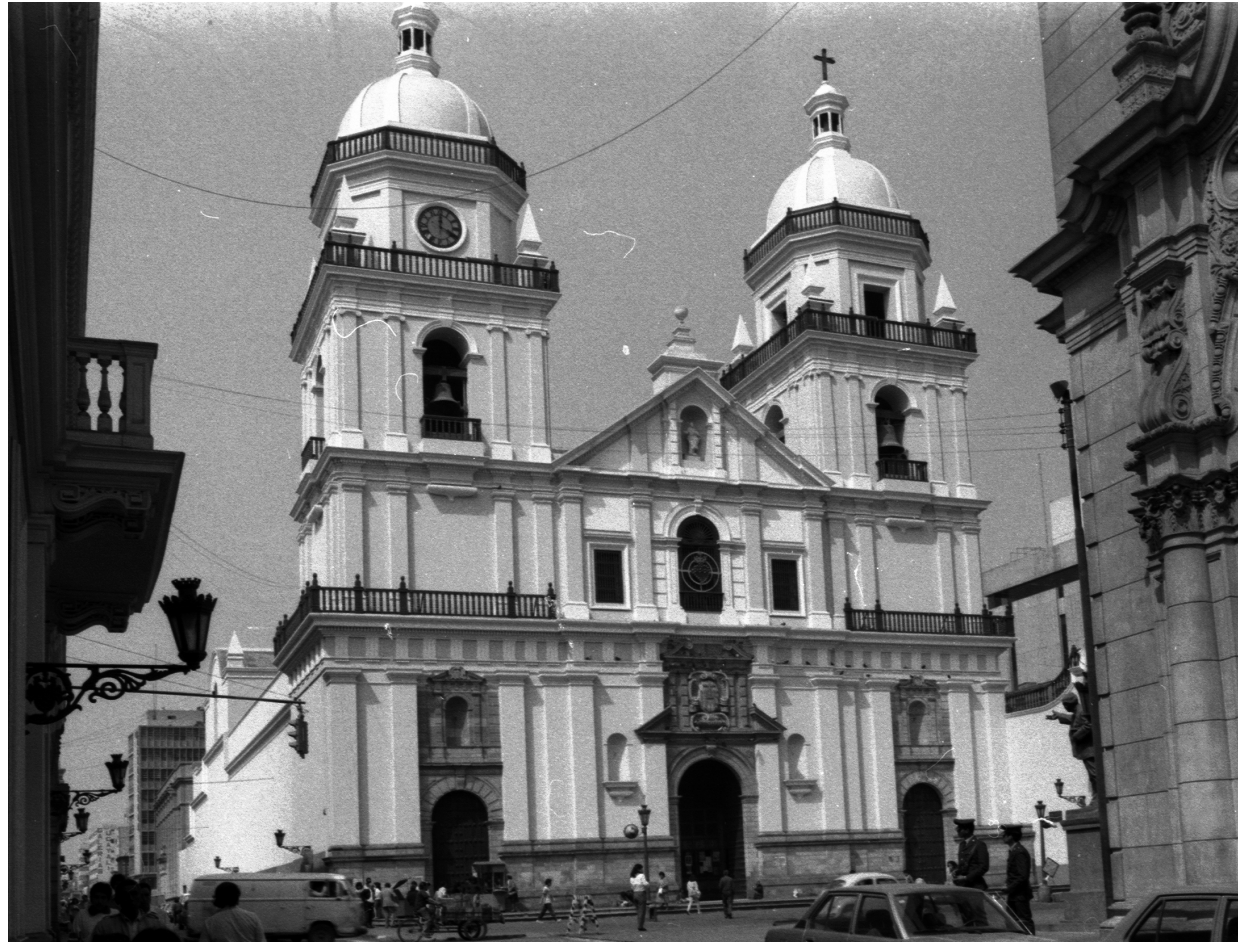

A partir de la revisión histórica de las intervenciones realizadas en el Centro de Lima, es posible determinar que estas comienzan aproximadamente en la cuarta década del siglo pasado. Ese primer periodo tiene como contexto inmediato el terremoto de 1940, que afectó numerosas edificaciones y propició diversos procesos de intervención en ellos, que se basaron en la experiencia del encargado de la obra, mas no en criterios técnicos específicos y/o históricos, pues en aquellos años prácticamente no existían escritos que sirvieran de referente o dieran luces sobre un método científico para intervenir edificios patrimoniales. Algunas intervenciones importantes de la época fueron la intervención de la portada de la Iglesia de La Merced realizada por el arquitecto Emilio Harth Terré en 1940 o la intervención en la Iglesia San Pedro realizada por el arquitecto Héctor Velarde en 1944 (Figuras 1y 2).

Este primer periodo culmina con la aparición de la Carta de Venecia en el año 1964, publicación a partir de la cual las intervenciones en el patrimonio edificado adquieren un matiz más científico, pues este documento plantea nuevos criterios que deben tomarse en cuenta. Paralelamente comienza con la capacitación de profesionales en el tema ${ }^{4}$, lo cual permitió incorporar una mayor conciencia en las intervenciones, lo cual da inicio a un nuevo periodo que dura hasta inicios de los años 90 . En este segundo periodo resaltan las intervenciones realizadas por los siguientes arquitectos:

\section{Arquitecto Víctor Pimentel Gurmendi}

- El proyecto de restauración y adecuación a nuevo uso de la Casona de San Marcos, ubicada en el Parque Universitario, entre los años de 1971 y 1973.

4. Es importante resaltar que conjuntamente con la llegada de la Carta de Venecia al Perú se funda en la Universidad Nacional de Ingeniería el Curso de Restauración de Monumentos a cargo del arquitecto Víctor Pimentel Gurmendi, uno de los cursos más antiguos de América, al igual que el que funda el Arquitecto Carlos Flores Marini en México. Ambos profesionales firmaron la Carta de Venecia.
Figura 1. Foto de la iglesia de San Pedro.

Fuente. Inventario del patrimonio monumental inmueble de Lima UNI Fundación Ford, 1988. 


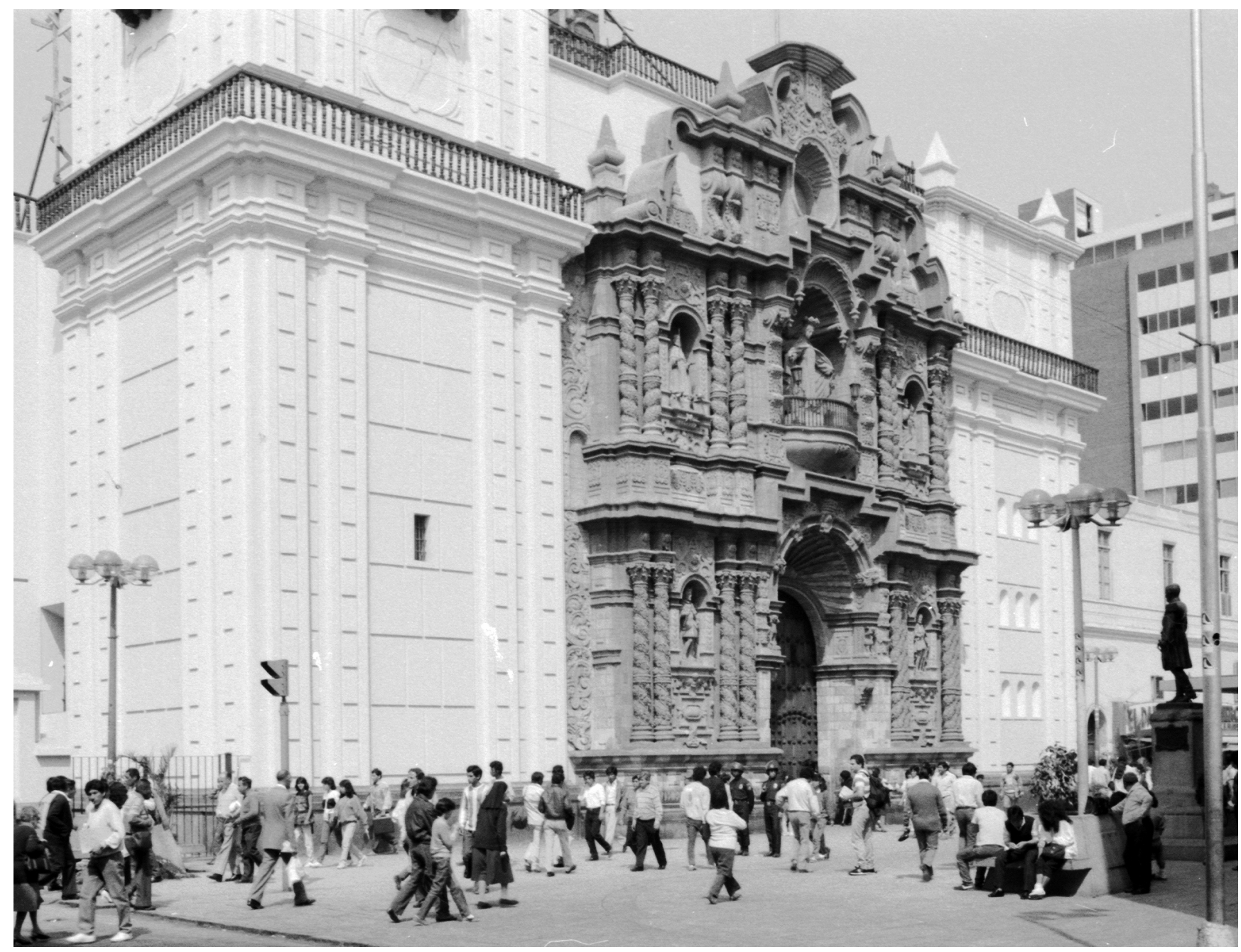

Figura 2. Foto de la iglesia de La Merced.

Fuente. Inventario del patrimonio monumental inmueble de Lima UNI Fundación Ford, 1988.
- El proyecto y la supervisión de las obras de restauración del Museo de Arte Religioso de la Catedral de Lima, ubicado en la Plaza Mayor de Lima, entre los años de 1973 y 1974.

- El Proyecto Integral para la Conservación, Restauración y Adecuación Museográfica del Conjunto Monumental de San Francisco de Lima, ubicado en el jirón Ancash, entre los años de 1974 y 1975.

- El Proyecto integral y supervisión de la restauración y adecuación a Nuevo Uso de la Casa Osambela, ubicada en el jirón Conde de Superunda, en el año de 1983.

- El Proyecto Integral y la Supervisión Técnica de la Obra de Conservación, Restauración y Adecuación a Nuevo Uso de la Casa de Ejercicios de la Orden Franciscana Seglar de Lima, ubicada en la avenida Abancay, en el año 1987.

\section{Arquitecto José Correa Orbegoso}

- La restauración de la Casa Jiménez, ubicada en la avenida Emancipación, entre los años de 1984 y 1985.

\section{Arquitecta Bertha Estela}

- La restauración de la Quinta de Presa, ubicada en jirón Chira, en el año 1985. 


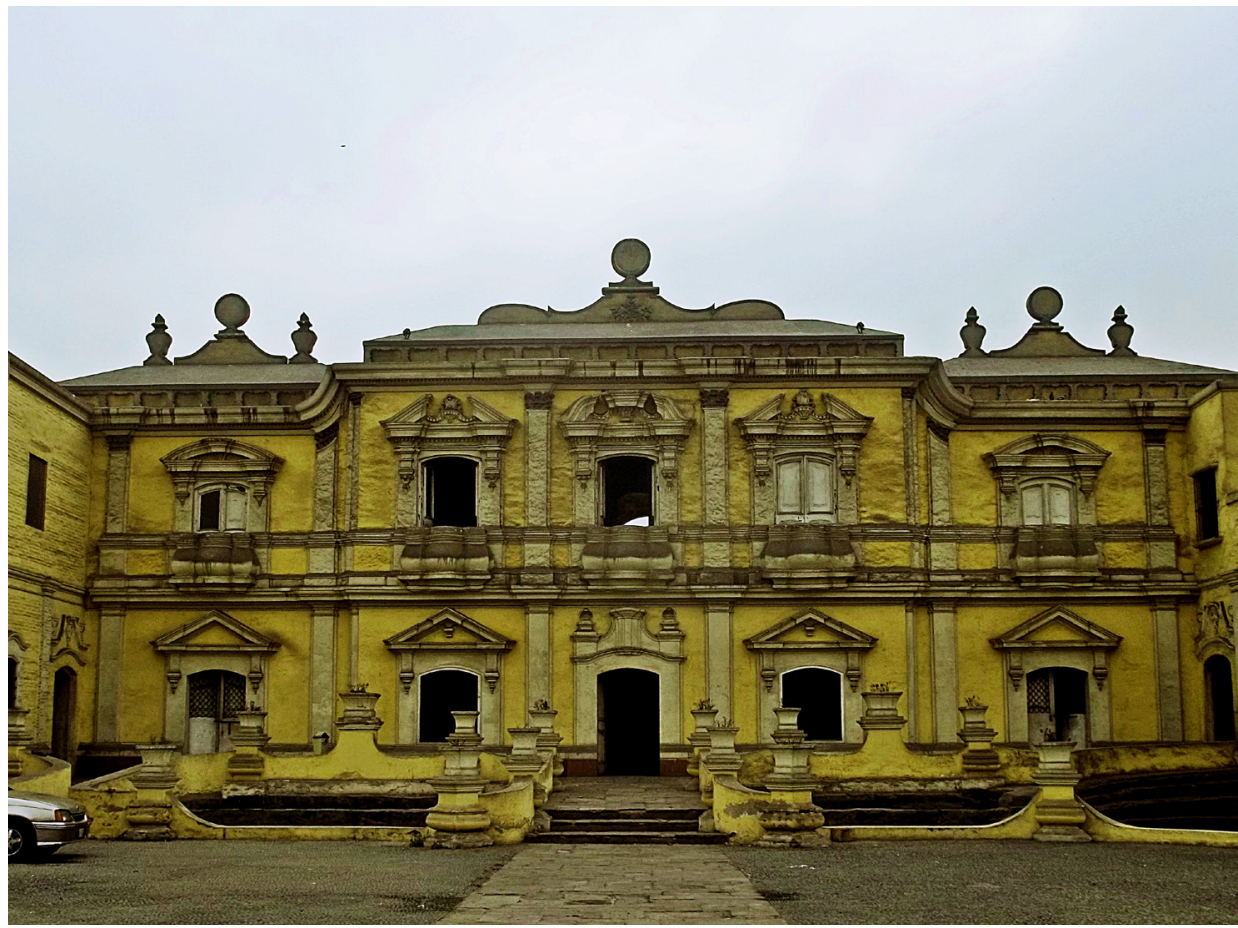

\section{Arquitecto José María Gálvez}

- Proyecto y obra de restauración del Museo de Numismática, en el año 1974

- Restauración del Pabellón Morisco del Parque de la Exposición, en 1988.

Es posible hablar de un tercer periodo, desde 1990 hasta 2010, en el cual tuvieron lugar hechos de suma importancia: en 1988 UNESCO declaró al Convento de San Francisco Patrimonio Cultural de la Humanidad, antecedente para que en 1991 se elabore la propuesta para UNESCO, realizada en conjunto por el Patronato de Lima ${ }^{5}$, la Municipalidad Metropolitana de Lima y el Instituto Nacional de Cultura, con la finalidad de incluir al Centro Histórico de Lima dentro de la lista del Patrimonio Cultural de la Humanidad ${ }^{6}$.

Dentro de este periodo es importante resaltar la contribución de la Agencia Española de Cooperación Internacional ( $\mathrm{AECl}$ ), mediante recursos que posibilitaron las intervención de edificios importantes; incorporaron a su accionar un primer acercamiento al tema de gestión patrimonial, y promovieron luego la formación de mano de obra calificada mediante la capacitación que brindan en las escuelas taller ${ }^{7}$.

5. El Patronato de Lima es constituido en 1993 como un órgano no gubernamental con características de institución cultural, y capacidad para desarrollar proyectos e investigaciones y solicitar recursos a nivel nacional e internacional.

6. El Centro Histórico de Lima fue inscrito tomando en cuenta el criterio de ser un ejemplo sobresaliente de un tipo de edificación o conjunto arquitectónico que ilustra una o varias etapas significativas de la historia humana, pues es un magnífico testimonio del desarrollo arquitectónico y urbanístico de una ciudad a lo largo del tiempo.

7. Las Escuelas Taller comenzaron sus actividades en el Perú a partir del año 1992, fecha en la que se apertura la Escuela Taller de Lima (ETL) en el marco del convenio firmado entre la Agencia Española de Cooperación Internacional (AECI) y el Instituto Nacional de Cultura del Perú (INC). La apertura de la ETL tuvo como objetivo la formación de jóvenes de escasos recursos en oficios vinculados a la conservación del patrimonio, a través de su participación directa en obras de intervención. Funcionó hasta su cierre en junio de 2012 y posteriormente reabrió un breve periodo de apenas unos meses en 2014 para volver a ser cerrada a inicios de 2015.

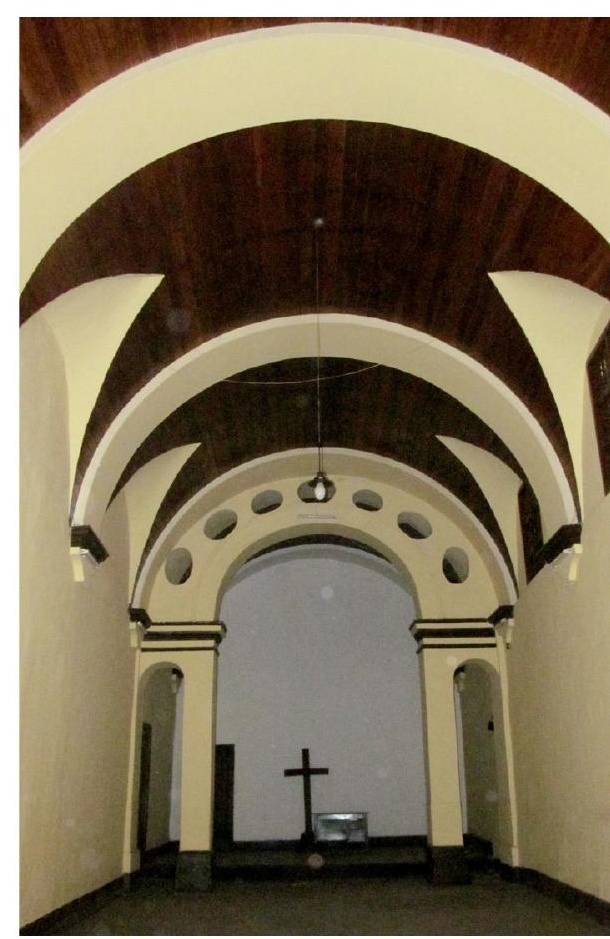

Figura 3. Fachada de la Quinta de Presa.

Fuente. Archivo María Copaira, 2012.

Figura 4. Foto interior de la Tercera Orden Franciscana.

Fuente. Archivo María Copaira, 2014. 
devenir Vol. 2, N³, ENERO- JUNIO 2015, PP. 63-78 - EstudIOS I ISSN 2312-7570

UNIVERSIDAD NACIONAL DE INGENIERÍA, LIMA

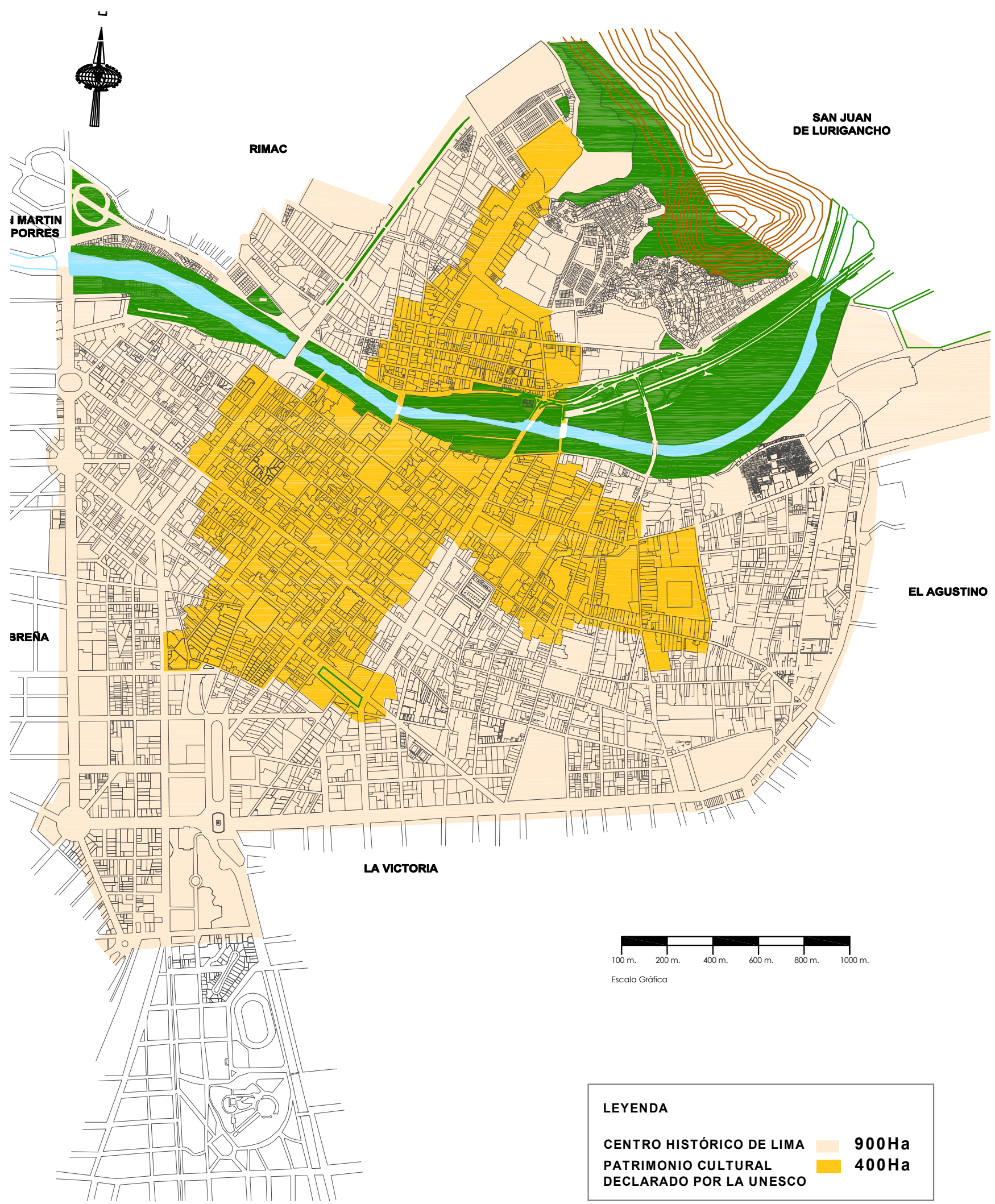

Plano 1. Zona del Centro Histórico de Lima declarada Patrimonio Cultural de la Humanidad.

Fuente. Elaboración propia María Copaira, 2015. 
Para el caso del Centro Histórico de Lima la obra más emblemática realizada durante esos años es la intervención de la Casona de San Marcos.

Durante la década de los 90 también se iniciaron los planes de recuperación del Centro Histórico de Lima, que en los años previos experimentó una serie de procesos que lo llevaron a su degradación, como los altos niveles de tugurización, el congestionamiento vehicular, la saturación, el sobreuso del espacio público, etc. Dichos procesos obedecieron principalmente a hechos económicos y sociales que suscitaron una "renovación urbana" improvisada. Esta aparición de nuevas edificaciones, y el cambio de usos de las existentes provocó la implantación y propagación de comercios dentro de las viviendas coloniales ${ }^{8}$, lo cual generó su división interna en varios usos (vivienda, comercios, depósitos, talleres, etc.). Dicha división tuvo repercusión en los aspectos legales de la propiedad de los inmuebles, ya estos que fueron subdivididos por sucesiones o herencias, para ser vendidos o arrendados, lo que creó confusas figuras legales de propiedad y/o tenencia. Desafortunadamente, los intentos del municipio para resolver estos problemas fueron insuficientes. Aunada a este fenómeno, la proliferación del comercio callejero fue otro detonante que agudizó la degradación del centro, pues se emplazó en las vías, apoderándose del espacio público e impidiendo el tránsito libre del peatón e, incluso, de vehículos, lo cual deterioró las calles.

La recuperación del espacio público en el Centro de Lima se inició a principios de 1996. Los primeros pasos se dieron durante el gobierno del alcalde Alberto Andrade Carmona, quien planteó por primera vez la recuperación del Centro Histórico de Lima dentro del plan de gobierno: se elaboró un plan maestro que tomaba como punto de partida la recuperación de los espacios públicos de la ciudad. El primer espacio público que se logró recuperar fue la Plaza Mayor de Lima, seguido de la intervención inmediata en la plaza San Martín y, posteriormente, en el Parque Universitario. Otros espacios recuperados durante esos años fueron la plazuela de Santo Domingo, la plazuela de San Agustín, la plazuela de Santa Clara, la plazuela de las Carrozas, la plaza de Monserrate, la plaza Castilla y la plaza Francia.

Se recuperaron también espacios de calles y avenidas que habían sido invadidas por el comercio informal; la primera intervención de este tipo se dio en el jirón Lampa, entre las cuadras siete y doce, que estaban ocupadas por cerca de 2000 comerciantes dedicados a la venta de artículos de ferretería y aparatos eléctricos, quienes fueron reubicados. Se remodeló las calles y se colocó mobiliario urbano nuevo, lo cual les dio una imagen nueva. Sin embargo, el caso más importante de recuperación de calles fue la reubicación de los comerciantes que se encontraban en los alrededores del Mercado Central: aproximadamente nueve manzanas estaban ocupadas por comerciantes informales que se habían apropiado de la calle, lo cual alteraba su configuración como espacio público. Una vez recobrada la zona, se iniciaron las remodelaciones y el retiro de elementos añadidos a la vía pública, como medidores de luz clandestinos.

Finalmente, cabe mencionar la recuperación de la zona de Polvos Azules, entonces atiborrada de comerciantes informales que fueron retirados del lugar para dar paso a la creación de un nuevo espacio con fin recreativo.

Además de la recuperación de espacios públicos, se buscó rescatar elementos que caracterizaran la arquitectura de los inmuebles. Con dicha intención se propuso el rescate de los balcones que, en un porcentaje alarmante de edificaciones, se encontraban en peligro de desaparecer debido al sobreuso y mal mantenimiento. Así, la

8. Principalmente predominó el uso comercial, particularmente de imprentas, lo cual actualmente sigue siendo una de las causas principales de degradación y pérdida de edificaciones patrimoniales. 


\begin{tabular}{lccc} 
Tabla 1. Tabla resumen de cifras del programa “Adopte un Balcón” \\
\hline Indicadores & \multicolumn{4}{c}{ Campañas } \\
\cline { 2 - 4 } & Primera & Segunda & Tercera \\
\hline Número de balcones & 47 & 24 & 1 \\
\hline Unidades inmobiliarias & 30 & 19 & 1 \\
\hline Empresas adoptantes & 40 & 24 & 1 \\
\hline Monto invertido (US\$) & $481,767.00$ & $224,823.00$ & $4,890.00$ \\
\hline Fuente: Elaboración propia María Copaira, 2015 & &
\end{tabular}

Municipalidad Metropolitana de Lima, como parte del Programa Municipal para la Recuperación del Centro Histórico de Lima (PROLIMA), comenzó a elaborar un levantamiento y registro de los balcones del Centro Histórico para posteriormente lanzar, a partir de agosto del año 1997, el programa Adopte un Balcón.

Realizando el análisis del alcance y ejecución de este programa, bajo las premisas antes mencionadas y tomando en cuenta los factores indicados, se pudo determinar cuáles fueron en primera instancia los criterios usados en la selección de los balcones en la primera y segunda campaña.

El primer criterio fue la ubicación, pues se consideraron aquellos que se encontraban en la Microzona A-I, parte de la Zona A, declarada como patrimonio cultural de la humanidad. El segundo estuvo vinculado a la categoría del inmueble (monumento histórico declarado, presunción monumental, ubicación en ambiente urbano monumental, etc.), y el tercer criterio empleado fue el estado de conservación en que se encontraba el bien inmueble a intervenir.

Finalmente, se tomó en cuenta para la selección el compromiso del balcón dentro del contexto urbano monumental y su valor artístico.

Siguiendo el modelo de análisis, se determinó cómo se llevaría a cabo el proceso de recuperación. En la etapa preintervención, se realizó la identificación de cada componente del balcón, su evaluación y la cuantificación del estado de deterioro, el análisis estructural, el levantamiento arquitectónico, el expediente fotográfico, la propuesta arquitectónica, el presupuesto base y el fichaje. Dicho trabajo fue realizado por el equipo formado por arquitectos de PROLIMA y estudiantes de arquitectura pertenecientes de la Universidad Nacional de Ingeniería (UNI) en el marco del convenio entre la Municipalidad de Lima y la universidad. En la etapa de intervención se llevó a cabo la ejecución del proyecto mediante la aplicación de diversos procedimientos especializados, llevando a cabo un registro fotográfico del proceso. Los alcances de cada proyecto eran diversos debido al estado particular de cada unidad inmobiliaria: algunos se encontraban más deteriorados y habían sufrido mayores alteraciones que otros. Finalmente, la etapa posintervención consistió únicamente en entregar a los propietarios un manual de mantenimiento.

El proceso de adopción de balcones se realizó de acuerdo a los siguientes pasos:

1. El adoptante debía presentar una carta a PROLIMA dando a conocer su decisión de participar en el programa, en la que indicara la ubicación el balcón de su preferencia.

2. Se suscribía un convenio entre la Municipalidad y el propietario con la finalidad de definir los compromisos y responsabilidades de cada una de las partes. 


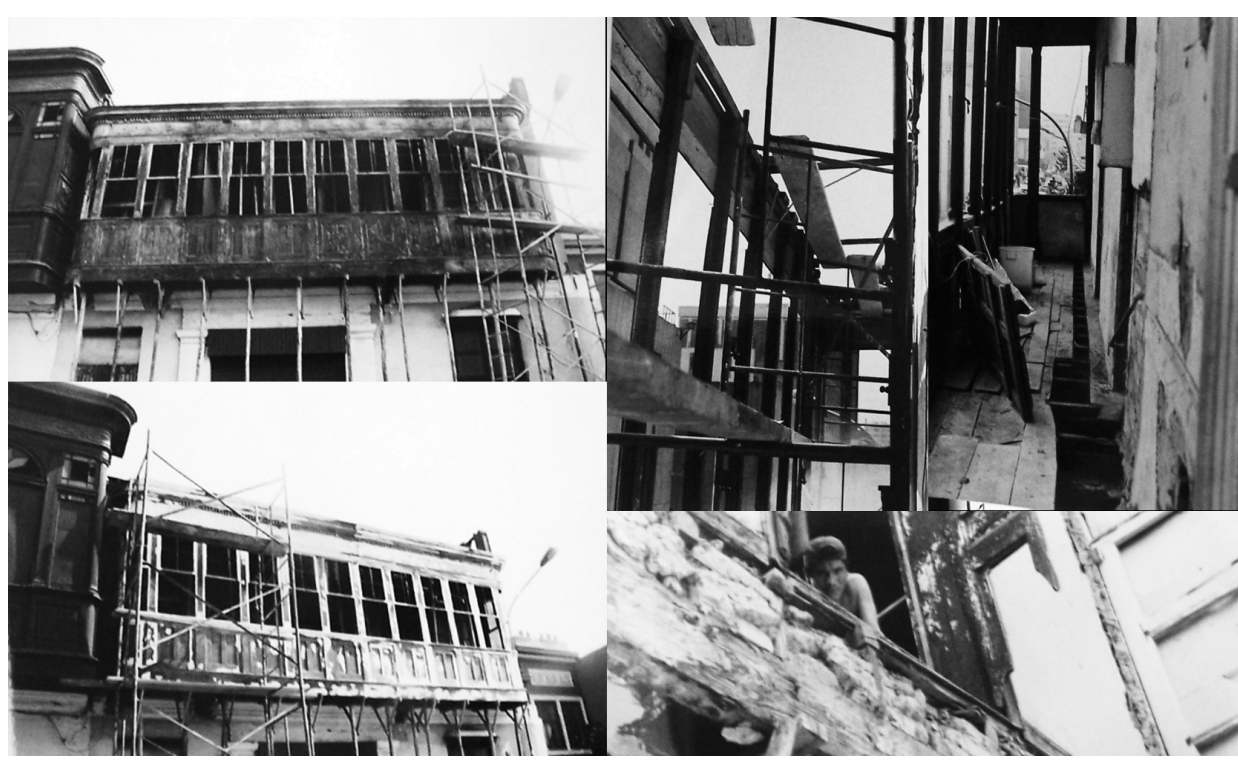

3. Una vez suscrito el convenio, la Municipalidad otorgaba al adoptante el expediente técnico del balcón. Así mismo, se le notificaba de modo escrito al propietario y los ocupantes de los trabajos de recuperación a realizarse.

4. Para efectos de encargar los trabajos de recuperación de los balcones, PROLIMA contaba con una cartera de especialistas y restauradores privados, los mismos que estaban a disposición de los adoptantes. En caso de que el adoptante contara con personal que pudiese realizar los trabajos de recuperación, estos debían acreditarse ante PROLIMA para garantizar que contaban con la experiencia suficiente para ejecutar los trabajos.

5. Una vez adoptado el balcón, PROLIMA designaba al profesional especialista a fin de hacer las coordinaciones y supervisión de los trabajos.

6. El adoptante estaba autorizado a colocar un cartel, de dimensiones establecidas en la Ordenanza 062-94 del Reglamento del Centro Histórico de Lima, en el que indicaba su participación en el programa, el mismo que permanecía durante el tiempo que durase la ejecución de los trabajos.

7. Finalizados los trabajos, la municipalidad colocaba una placa de reconocimiento con el nombre del adoptante.

Existieron las siguientes tres campañas de adopción:

- En la primera campaña, que comenzó en agosto del año 1997, se intervinieron los balcones que estaban ubicados en la microzona A-l, que comprendía el área entre las avenidas Tacna y Abancay, y los jirones Cusco y Emancipación, hasta el rio Rímac, donde se ubicaban aquellos balcones con más alto riesgo de colapso. En esta primera etapa se consiguió la adopción de 47 balcones, distribuidos en 30 unidades inmobiliarias, lo cual implicó una inversión total de US\$ 481,767.00.

- En la segunda campaña, que comenzó en abril del año 1998, se intervinieron los balcones que se ubican en la microzona A-III, comprendida por las siguientes vías: la Av. Tacna, el Jr. Moquegua, el Jr. Ocoña, el Jr. Apurímac, el Jr. Puno y el Jr. Rufino Torrico. En total se logró la adopción de 24 balcones, distribuidos en 19 unidades inmobiliarias, lo cual requirió una inversión total de US\$224,823.00.

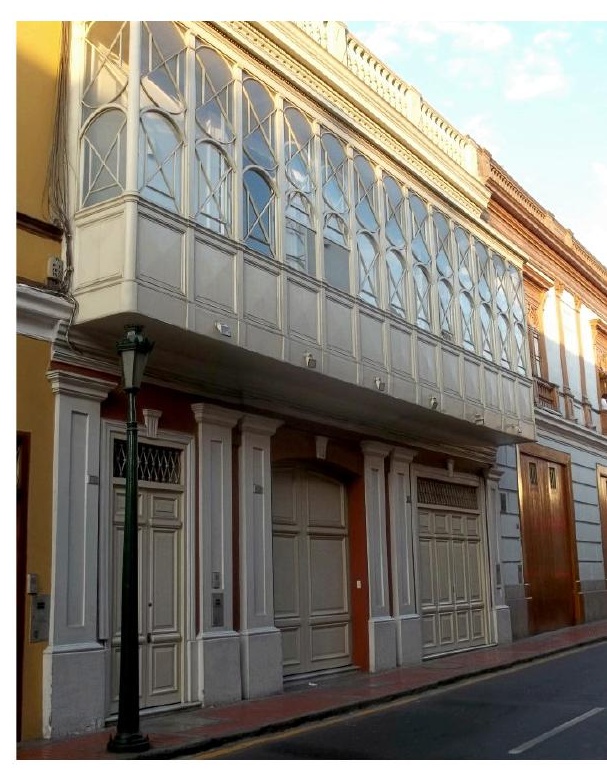

Figura 5. Fotos de uno de los balcones que fue intervenido.

Fuente. Archivo de PROLIMA, 2015.

Figura 6. Fachada de uno de los inmuebles intervenidos. Jr. Ancash cdra. 2 - 2012.

Fuente. Archivo María Copaira, 2012. 
devenir Vol. 2, N³, ENERO- JUNIO 2015, PP. 63-78 - EsTUDIOS I ISSN 2312-7570

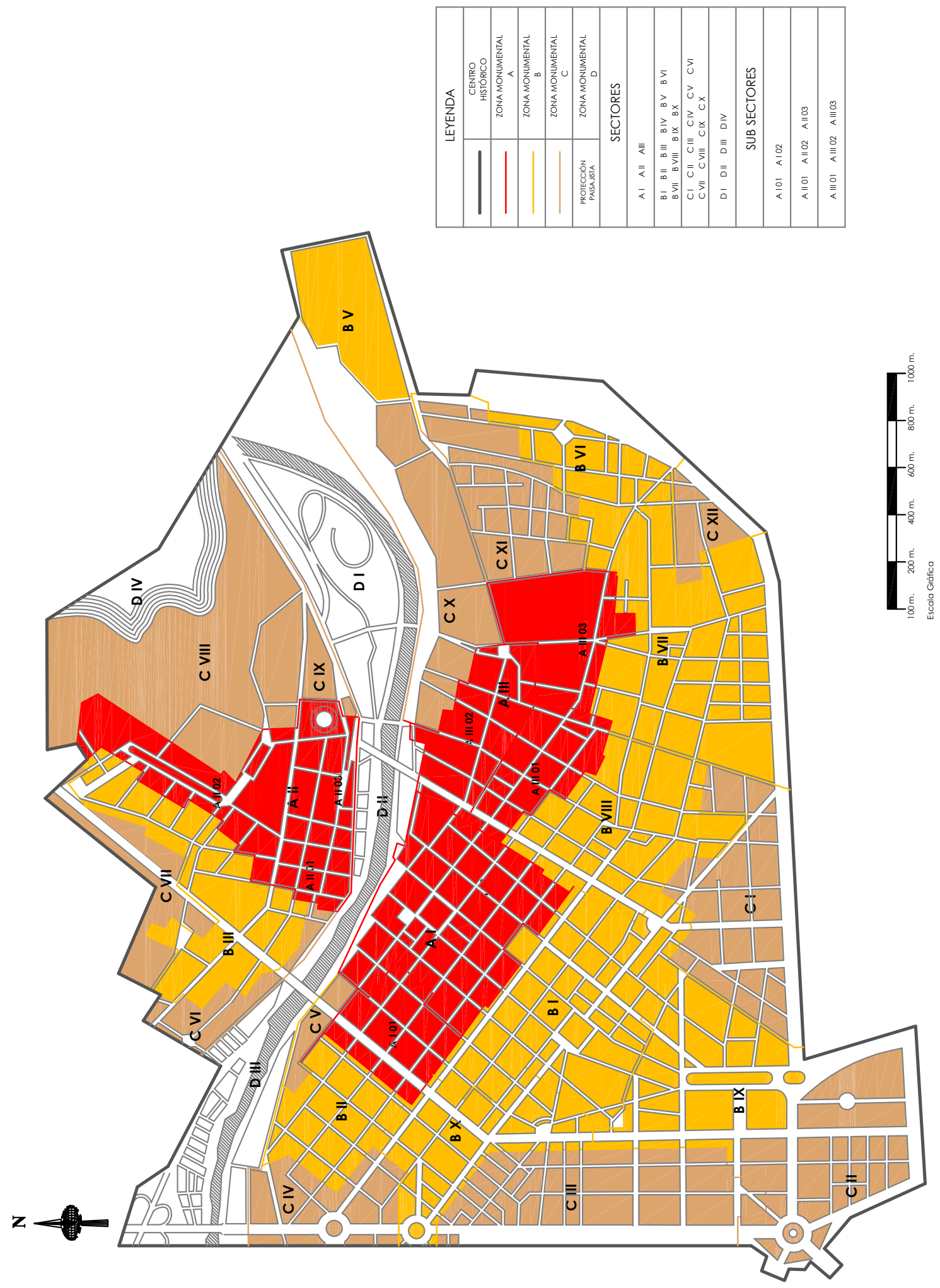

Plano 2. Plano de estructuración del Centro Histórico de Lima9.

Fuente. Elaboración propia María Copaira, 2015.

9. El plano de estructuración mostrado corresponde al que se encuentra en el Reglamento del Centro Histórico de Lima desarrollado en el año 1991 por la Municipalidad Metropolitana de Lima, el Patronato de Lima y el Instituto. 
- La tercera campaña, que comenzó en junio del año 2002, fue la última del programa y tuvo menor acogida que las antecesoras, pues solo se realizó la adopción de un balcón, a pesar que se había realizado el empadronamiento de 15 balcones ubicados en el distrito del Rímac, pertenecientes a la microzona A-II del Centro Histórico de Lima. La inversión total fue de US\$ 4,890.00.

Podríamos afirmar que el programa Adopte un Balcón tuvo éxito relativo, pues si bien en las primeras dos campañas se logró la adopción de un considerable número de balcones (71 en total), se fracasó en la aplicación de una metodología adecuada que considerase los diversos factores externos sociales y económicos, y de un plan de gestión que hiciera sostenible la recuperación. En la mayoría de casos no existió un seguimiento posterior tras finalizar las obras; muchos de los propietarios dejaron el mantenimiento de lado, y volvieron a dotar de un uso inadecuado a los balcones, lo cual generó nuevamente su degradación y, en algunos casos, su desaparición (Figuras 5 y 6).

En síntesis, a modo de conclusión, es posible afirmar que uno de los principales problemas es que en la actualidad la polémica y el debate sobre las intervenciones (Ilámese conservación, restauración, ampliación, consolidación, etc.) se ha centrado en aspectos técnicos, y problemas netamente de índole operativo y material, lo cual responde a la obvia interrogante de cómo se interviene una edificación patrimonial, pero deja de lado aspectos fundamentales, como los que surgen como respuestas al cuestionamiento de para qué se interviene, lo cual remarca la falta de uso de modelos de gestión que enlacen todos los aspectos necesarios en el manejo de las intervenciones sobre el patrimonio edificado para lograr que estas sean sostenible y perdurable a través del tiempo.

Con el transcurso de los años, indudablemente se han mejorado ampliamente las técnicas de intervención orientadas a la conservación, restauración y consolidación gracias a las mejoras en la capacitación técnica actualmente disponible en nuestro medio; sin embargo, apremia una reflexión sobre la necesidad que motiva a trabajar en la recuperación del patrimonio edificado. Dicha reflexión es crucial para la toma de conciencia sobre los "criterios" de intervención, ya que debería ser un medio para profundizar lo más posible en el conocimiento de aquello que se va a intervenir y tomar en cuenta cada factor a fin de plantear soluciones integrales que partan de la percepción de la edificación como un objeto inserto en un contexto urbano y social para así poder discernir sobre cuál es la opción más adecuada de intervención.

\section{Referencias}

Alonso, M. (1992). El patrimonio histórico: Destino público y valor cultural. Madrid, España: Civitas. Ballart, J. (1997). El patrimonio histórico y arqueológico: Valor y uso. Barcelona, España: Ariel.

Ballart, J. y Juan, J. (2001). Gestión del patrimonio cultural. Barcelona, España: Ariel.

Banco Interamericano de Desarrollo (2001). Una obra en marcha. El Banco Interamericano de Desarrollo y la protección del patrimonio cultural. Washington D.C., EE.UU.: Autor.

Brandi, C. (1988). Teoría de la restauración. Madrid, España: Editorial Alianza.

Consejo Internacional de Monumentos y Sitios (ICOMOS). (1964). Carta de Venecia.

Carrión, F. (Ed.). (2001). Centros históricos de América Latina y el Caribe. Quito, Ecuador: Facultad Latinoamericana de Ciencias Sociales (FLACSO) Ecuador.

Capitel, A. (1988). Metamorfosis de monumentos y teorías de la restauración. Madrid, España: Alianza.

Ceschi, C. (1970). Teoria e storia del restauro. Roma, Italia: Mario Bulzoni.

Choay, F. (1997). Alegoría del patrimonio. Barcelona, España: Gustavo Gili.

De Gracia, F. (2001). Construir en lo construido: La arquitectura como modificación. Madrid, España: Nerea. 
devenir Vol. 2, N³, ENERO- JUNIO 2015, PP. 63-78 - ESTUDIOS I ISSN 2312-7570

UNIVERSIDAD NACIONAL DE INGENIERÍA, LIMA

De Solá-Morales, I. (2006): Intervenciones. Barcelona, España: Gustavo Gili.

Fernández, J. (1996). Introducción a la conservación del patrimonio y técnicas artísticas. Barcelona, España: Ariel.

Hayakawa, J. (2001). Restauración en Lima: Pasos y contrapasos (Tesis de investigación de pregrado). Universidad Nacional de Ingeniería, Lima, Perú.

García, N. (1987). ¿Quienes Usan el Patrimonio?: Políticas Culturales y Participación Social. Antropología (Boletín del Instituto Nacional de Antropología e Historia) 15/16(julio - octubre) 1987, 11-23.

Norberg-Schulz, C. (1975). Nuevos caminos de la arquitectura: Existencia, espacio y arquitectura. Barcelona, España: Blume.

Real Academia Española (RAE) (2011). Diccionario de la Lengua Española. Madrid, España: Espasa.

Rivera, J. (2001). De Varia Restauratione. Teoría e Historia de la Restauración Arquitectónica. Madrid, España: Editorial América Ibérica. 